\title{
La acción de José Patiño en Cádiz y los proyectos navales de la Corona del siglo XVII
}

\section{ANA CRESPO SOLANA}

La' obra de José Patiño como estadista, diplomático y economista ha sido señalada en varias ocasiones, aunque no profundizada en un estudio que nos ofresca una visión más concreta y pulida.

Su aparición en la escena política española durante el reinado de Felipe V se produjo unido en fortuna a otra persona, el Almirante Don Andrés de Pez, gobernador del Consejo de Indias en 1717 y posteriormente primer Secretario de Guerra y de Marina, cuando esta institución se creó por segunda vez en 1721 (1). La actividad política de José Patiño está, en cierto modo, conectada con las inspiraciones del Almirante, como el propio Patiño explica en su "Exposición Reservada" (2). En 1726, y tomando la dirección de la Secretaría de Marina e Indias, Patiño aparece en la Administración central, acaparando después casi la totalidad del poder como primer ministro de Felipe V. En la escasa bibliografía de que disponemos sobre su gobierno se apunta que, como hombre de Estado, Patiño supo calibrar el papel de España en el nuevo equilibrio internacional, y emparejar éste con los intereses de la nación (3).

Como muy bien ha resumido Ravina Martin (4), Patiño se encargó fielmente de las cuiestiones de política comercial y naval que tenían como objetivo primero el puerto de Cádiz, aunque hay que indicar que sus actividades entroncan con

(1) Cfr: GIRARD, Albert, La rivalité commerciale et maritime entre Seville et Cadix jusqu' a la fin du XVII siècle, Burdeos, 1932; y algunas notas sobre el gobierno personal de D. Andrés de Pez como Secretario de Guerra y Marina en: BERNARD, Gildas, Le Secrétariat d'Etat et le Conseil espagnol des Indes (1700-1808), Geneve, Paris, 1972, pp. 12, 33, 35, 47 y 78-112.

(2) RODRIGUEZ VILlA, A., Patiño y Campillo. Reseña biogräfica de estos dos ministros de Felipe $V$, Madrid, 1882, pp. 28-69, y lo explica también RAVINA MARTIN, M, El Pleito Cádiz-Sevilla por la Casa de la Contratación. El Memorial de Francisco Manuel de Herrera, Cádiz 1984, pp. 65 y 66.

(3) RAVINA MARTIN, M., Op. Cit, p. 64. Bibliografía sobre Patiño: BETHENCOURT MASSIEU, A., Patiño en la política internacional de Felipe $V$, Valladolid, 1954, RODRIGUEZ VILLA, A., Op. Cit, ESCUDERO, J.A., Los origenes del Consejo de Ministros, Madrid, 1979, Tomo I, pp. 84 y 89-98.

(4) RAVINA MARTIN, M., Op. Cit, pp. 66-70.

DOI: http://dx.doi.org/10.25267/Trocadero.1995.16.02 
algunas otras que se realizaron a primeros de la centuria por otros ministros de Felipe $\mathrm{V}$ en el contexto reformista que se respiraba (5).

Una de sus realizaciones más importantes hace referencia al puerto gaditano y el intento de habilitación de la zona de la bahía como sede de una auténtica base naval y militar que sirviera de clave a la Carrera de Indias.

Generalmente se ha hecho hincapié, y con importantes razones,en la obra de Patiño como bienhechor de la Armada española. En 1724, inició la construcción en Cádiz del arsenal de la Carraca, y durante los años que rodean esa fecha encontramos al ministro muy preocupado por convertir en un verdadero centro marítimo-militar a esta zona de la bahía. Entre 1717 y 1730, Patiño creó la Compañía de Caballeros Guardias marinas, redactó unas Ordenanzas provisionales de la Armada y unas instrucciones para arsenales. En 1725 redactó las Ordenanzas sobre Cuenta y razón (probablemente a imitación de Francia). Patiño se encargó personalmente de la institución Casa-Intendencia con amplísimas funciones que dirigía la navegación y el comercio, en un intento de reorganizar las viejas disposiciones que emanaban de la antigua Casa de la Contratación de Sevilla. Tanto para una y otra cuestión actuó como árbitro y director, y aunque sus actuaciones parecen prudentes y conservadoras con respecto a las actividades mercantiles, y sobre todo hacia las instituciones más representativas del comercio (como el Consulado), sí fue bastante innovador con respecto a la navegación atlántica, propiciando la construcción naval con la ayuda de asentistas españoles y extranjeros, y ofreciendo un mayor dinamismo administrativo al movimiento portuario (6). Por último, entre esos años y 1726 se crearon los astilleros de Puntales y Matagorda, finalizando así su actuación directa en la preparación del puerto gaditano para su conversión definitiva en Departamento Marítimo (7).

Sabemos muy poco sobre la línea de pensamiento que siguió el ministro en sus quehaceres en Cádiz (si es que hubo una línea de pensamiento personal). Sería también una buena información, si por nuestra parte pudiéramos calibrar algunas de las ideas confeccionadas por Patiño en esos años y que le sirvieron como base experimental para sus posteriores decisiones, cuando, después del cambio político entre 1725 y 1726 , acaparó todo el poder gubernamental como ministro de Felipe V.

Como fuentes posibles contamos con dos textos escritos supuestamente o redactados por el propio Patiño ("Exposición Reservada" y su voto en las Juntas de 1722 y 1723). En segundo lugar, y aunque sujeto a reconstrucción, queremos resaltar algunas de las actividades concretas que personalmente, y en compañía de otras entidades e individuos, realizó como Intendente-presidente en la bahía

(5) PEREZ-MALLAINA BUENO, P.E., Politica naval española en el Atlántico. 1700-1715, Sevilla 1982, y CRESPO SOLANA, A., La Casa de la Contratación y la Intendencia General de la Marina en Cádiz en el contexto reformista del reinado de Felipe V, 1717-1730, Tesina de licenciatura leída en la Universidad de Cádiz, junio de 1994, pp. 30-39.

(6) CRESPO SOLANA, A., Op. Cit, pp. 75 y ss. Sobre la vertiente economista de José Patiñoexisten algunos trabajos: RUIZ RIVERA, J.B., "Patiño y la reforma del Consulado de Cádiz en 1729", Temas Americanistas, 5, Sevilla, 1985, pp. 16-21.

(7) El proceso ha sido analizado en su fase final por: TORREJON CHAVES, A., La Nueva Población de San Carlos en la Isla de León. 1774-1806, Min. de Defensa, Madrid, 1992, 2 vols. 
gaditana, y que lo relacionan claramente con el poder local gaditano. En ello contó desde la propia Administración central, con un apoyo especial por parte de Andrés de Pez. La colaboración e interes de ambos se ve claramente durante el pleito que tuvo lugar en la Corte por el traslado de los tribunales a Cádiz desde su antigua sede sevillana.

\section{ALGUNAS OBSERVACIONES SOBRE EL PAPEL DEL ALMIRANTE PEZ Y IAA IDEA DE UN PROYECTO NAVAL}

El papel que Andrés de Pez realizó desde la propia Administración central en lo referente al puerto y bahía de Cádiz la intuímos a través de las propias Actas del Cabildo gaditano entre 1717 y 1723. Los propios miembros del Cabildo decidieron contar con este principal apoyo cuando se convocaron las Juntas de Madrid de 1722 y 1723, y decidieron enviar, provocando las quejas del Consulado sevillano, al representante Francisco López de Villamil (8). Debido a la problemática situación en la que se desarrolló el cambio de tribunales entre las dos ciudades y toda la compleja trama que este hecho llevó aparejada, todas las conclusiones extraídas de aquellos acontecimientos son partidistas y, no con falta de fundamento, presentan a algunos de aquellos sucesos como un auténtico montaje político (9).

Indudablemente actuaron unas posibles esferas de poder; que intervinieron en toda la evolución teórica y práctica del proceso hasta 1725, y unos grupos de presión que, bien directamente o desde la sombra, ayudaron, en definitiva, a la culminación de la causa. Otros factores externos ayudaron, como fue el cambio político de 1725 y 1726 en que se produjo una renovación en el poder ministerial, provocando, entre otras cosas, la subida de José Patiño al gobierno de Madrid. Sin embargo, no podemos dejar de considerar la indiscutible conexión que el proyecto aplicado a la bahía de Cádiz tiene con los amplios debates sobre la reforma de la marina y el comercio que tuvieron lugar en la Corte desde finales del siglo XVII (10). Los ministros que integraron el gobierno de Felipe V en las dos primeras décadas de la centuria se vieron inmersos en argumentos diferentes sobre la misma política, y en gran medida, algunos de ellos se convirtieron en depositarios y conservadores de ideas anteriores sobre la necesidad de establecer,

(8) Archivo Histórico Municipal de Cádiz. (AHMC) Libros de Actas Capitulares, (L.A.C) Cabildo del 11 de diciembre de 1722, Carta de Andrés de Pez a la ciudad para que en la Corte se contradigan las gestiones hechas por Sevilla. El Cabildo acuerda hacer llegar una representación al rey a través del ministro. Se decidió por parte del Cabildo y algunos comerciantes de la ciudad, como fue D. Andrés Martínez de Murguía, el envío de Francisco López de Villamil. La relación entre Martínez de Murguía y Pez queda probada por CASTRO, Adolfo de, Vida del Almirante D. Andrés de Pez, Ministro de Marina, Cádiz 1879, pp. 20-29.

(9) Bibliografía sobre la rivalidad Cádiz-Sevilla, Cfr: RAVINA MARTIN, M., Op.Cit, y GIRARD, Op. Cit.

(10) A pesar de lo mucho que se ha debatido, los artículos más completos que hemos podido localizar sobre el tema son: CALLAGHAM, W.J., "A note on the Real y General Junta de Comercio. 1679-1814", Economical Historical Review, (E.H.R), 21, London, (1986), pp. 519-528, y MOLAS RIBALTA,P, "La Junta de Comercio y Moneda", Cuadernos de Historia Anexos de la Revista Hispania, 9, Madrid, (1978), pp. 1-39. 
con ayuda de un programa sistemático, unas bases navales (por no decir policiales) y de control aduanero en zonas claves de la geografía europea (11).

Uno de los ministros que se dejaron influir por éstas ideas reformistas fue Andrés de Pez. Aunque el Almirante murió en marzo de 1723 (dos años antes de la decisión oficial definitiva), debemos de considerar algunas de las gestiones que realizó por la ciudad y de las cuales Patiño se considera heredero y continuador (12). Pero lo que pudieron ser ideas personales se entrelazan con otros factores: en primer lugar destacamos su relación con los poderes locales gaditanos y con un sector concreto, armadores, marinos y comerciantes (13). Según los propios comerciantes de la ciudad, la Real Cédula del traslado de la Contratación e instalación de la Intendencia General de la Marina en la ciudad en 1717 fue una decisión personal de Pez (14). El último deseo del Almirante y gobernador del Consejo de Indias fue que se debía volver a estudia el Informe que D. Manuel García de Bustamante, miembro del citado Consejo, presentó el 1 de octubre de 1701 (15). ¿Aquí vemos como el ministro es heredero de, lo que podríamos llamar, una corriente de pensamiento?.

En los últimos meses de su vida, Andrés de Pez hizo todo lo posible para reunir la mayor parte de documentos favorables a la utilización del puerto de Cádiz con la intención de que éstos contrarrestaran los memoriales sevillanos y a los opositores del propio Consejo, que indicaban la conveniencia que presentaba la llegada de las flotas a la bahía, y que es el informe más completo que existe referente a los antecedentes del traslado que se vieron en la Junta de Madrid de 1722 (16).

La revisión del memorial de Bustamante es lo único de que disponemos para fijar la postura del Almirante con respecto a los cambios practicados en la cabecera de las flotas y a su intención de fijarla en el puerto de Cádiz, postura que Patiño continuaría después como Secretario del Despacho a partir de 1726 (17). Hoy sabemos que este memorial es un copia casi exacta de otro proyecto presentado en las Juntas que tuvieron lugar durante los años 60 y 70 del siglo XVII, sobre la necesidad

(11) Dentro de un contexto mucho más amplio esta idea está latente en España desde tiempos del Gobierno de Olivares. En Europa había, en el mar, dos arterias principales del comercio europeo que el Imperio de los Habsburgo, y sus enemigos, deseaban controlar, ya sea por bases estratégicas, aduanas, guardacostas o por establecimiento de bases de Armadas: el Canal de la Mancha y el Estrecho de Gibraltar. Esta idea la vemos recogida en STRADLING, R.L., La Armada de Flandes, Politica naval española y guerra europea, 1568-1688, Madrid, 1992, p. 49.

(12) CASTRO, Adolfo de, Op. Cit., p. 29.

(13) Ibidem.

(14) AHMC. L.A.C, Tomo 76 (1720), p. 28 y GIRARD, A., Op. Cit., pp. 79-80.

(15) Archivo General de Indias. (A.G.I) ARRIBADAS 192, Carta escrita por Pez a Patiño en 1722. También figura en el Informe hecho después de su fallecimiento cuando la Junta se reunió por segunda vez en 1723 .

(16) A.G.I. INDIFERENTE GENERAL, 2020. Se mandó hacer por D. Miguel Fernández Durán por orden del 7 de marzo de 1720 .

(17) El Informe de D. Manuel de Bustamante es citado por primera vez en la obra de GIRARD, A., Op. Cit., p. 21, como una de las primeras ocasiones en que se menciona la unión de los cambios practicados a la marina y al comercio y su relación con el puerto de Cádiz. Informe que hemos utilizado es la copia que pasó a la Junta para el Restablecimiento del Comercio el 13 de febrero de 1706. A.G.I. INDIFERENTE GENERAL, 2046, A. 
de establecer una Compañía Universal de las Indias con sede en un puerto español que compitiese con el resto de las compañías comerciales creadas en Europa (18).

$\mathrm{El}$ análisis de este documento denota dos cuestiones muy importantes. En primer lugar demuestra que hubo un programa coherente de actuación en este campo de introducir algunas innovaciones referentes al comercio y la navegación hacia las Indias que mejorara, aún dentro del Mercantilismo, las bases del monopolio. Este programa, que parte de finales del siglo XVII, y que se vería materializado en las varias juntas de comercio que tuvieron lugar desde 1679, englobaría también a las primeras reformas emprendidas durante los ministerios de Tinajero y Alberoni, primeros reformadores de la Armada y el comercio en el siglo XVIII (19).

En segundo término, en los debates relativos a la creación de una base naval-militar y comercial se contempló la posibilidad de colocar esta base en el puerto de Cádiz por las razones que, a parte de ser especificadas en éste y otros memoriales sobre las tan esperadas reformas, se verán materializadas coherentemente por el propio Patiño durante su estancia en la ciudad entre 1717 y 1726. El contenido de este memorial hace referencia, en primer lugar, a la creación de una Compañía Universal para las Indias con base en un puerto que turviese las características adecuadas a ser también una zona proveedora de material naval. En el memorial se vislumbra las influencias que este proyecto recibió de las compañías fundadas en otros países europeos desde la segunda mitad del siglo precedente, y en cierto modo, hay una razón lógica por la que la Corona española plantease también la posibilidad de la creación de este tipo de compañias: casi todos los Imperios coloniales estaban bajo la administración de una Compañía Universal de Comercio, a excepción de las colonias españolas de América (20).

De entre todos los postulados que deseaba proponer este proyecto, uno de los más interesantes era que en ella tendría cabida los negocios de los comerciantes españoles, pero también se aceptaría los de los "extranjeros, amigos parciales y aliados", quienes para Bustamante eran los flamencos y los italianos. ¿Este proyecto propone la inclusión de los extranjeros?. Un punto interesante, y relacionado con lo anterior, era una cierta perseverancia en incluir, por todos los medios posibles, a la producción nacional española (seda y lanas, incitando a la participación de los gremios) junto con una admisión racional de mercancías traídas desde otros países europeos (21).

Es obvio que se consideraba la realidad del puerto gaditano y su especial configuración. Este proyecto introducía otras novedades interesantes como la inclusión del tráfico organizado hacia otros puertos americanos: Buenos Aires, Honduras, Campeche, Venezuela, Costa de Tierra Firme, etc. Como sabemos este deseo será uno de los mayores logros de la nueva centuria. Con dinero de los

(18) Cfr: ALEJANDRE GARCIA, A., "Un Proyecto de Compañía Universal del Comercio con las Indias en el siglo XVII", Actas y Estudios del III Congreso del Instituto Internacional de Historia del Derecho Indiano, Madrid, (1973), pp. 925-984.

(19) MOLAS RIBALTA, P., Art. Cit., En estas Juntas participaron entre otros Orry, Daumberton y Bustamante. Según el autor también contaba con la presencia de hombres de negocios "representantes de las ciudades comerciantes del reino", p. 16.

(20) A.G.I. INDIFERENTE GENERAL, 2046 A, Memorial de Bustamante, Passim.
(21) Ibidem. 
fondos de esta compañía se llevaría a cabo la compra de provisiones, y los pagos de "gente de mar" de los navíos, es decir, se especifica todo lo concerniente al mantenimiento de una Armada.

La propuesta recogía además un punto relativo al problema de las aduanas y al control del oro y la plata, para que ésta se quedase en España, una vez pasados los registros de los puertos de Cádiz y Sanlúcar. La cuestión fiscal aparece como intrínsecamente unida a la utilización del puerto de Cádiz: el memorial proponía que las flotas y Galeones debían dar fondo en la bahía gaditana y da una respuesta muy lógica. Las flotas, cuando llegaban desde América, lo primero que se encontraban era precisamente la zona de la bahía, donde tenían lugar también los fraudes y robos, luego era alli donde debía de ponerse el mayor remedio, no en Sevilla que estaba río arriba, y cuando llegaban los barcos a hacer el registro de vuelta se había liquidado la mitad de su preciada carga.

En algunos puntos del memorial se exponen las líneas de la subvención para la fábrica de bajeles, astilleros y el suministro de Armadas. En ello se indicaba cómo debía realizarse la construcción y mantenimiento de astilleros en la Península y en América, enviándose desde los primeros a los segundos todo el material necesario para la construcción que allí faltaba: hierro, jarcias, lona, etc. La Corona debía de considerar estos gastos como de primera necesidad porque así no sólo se aseguraba el comercio, sino que también se vigilaba el movimiento portuario a nivel comercial y militar (22).

Andrés de Pez presentaba la revisión de este memorial como uno de sus propios argumentos pero el Almirante y Ministro de Marina era consciente de la infraestructura que era necesario para la realización de un proyecto semejante, y lo que es más sabía que este plan implicaba la colaboración de comerciantes, marinos, armadores de buques y hombres de negocios. Ello explica su colaboración con el Cabildo gaditano y con algunos marinos de la ciudad.

No sabemos hasta qué punto el proyecto de Pez y Patiño conllevaba exactamente la creación de una Compañía Universal con base en la ciudad gaditana, lo que sí es cierto es que muchos de los puntos desglosados en él fueron llevados a cabo en el puerto de Cádiz cuando se unieron las competencias de la Contratación a la recien creada Intendencia de Marina en 1717.

La Administración consideró largamente toda esta serie de proyectos materializados en las Juntas de Madrid de 1722 y 1725 . En cierto modo, conociendo el contenido de éstos, podemos vislumbrar el por qué la Corona no llegó a cumplir el Decreto de revocación y traslado de los tribunales a Sevilla en 1725. Dos factores podemos resumir de los debates: el puerto de Cádiz se había estado preparando estructural y económicamente; y, en segundo lugar, la propia Corona había manifestado esta inclinación ya desde la segunda mitad del siglo XVII, en vista de las reformas para la marina y el comercio que se respiraban. Existe un documentos anónimo que insiste en las especiales características de la zona como puerto natural, propicia a la construcción de una base naval (23).

(22) Ibidem, Cfr: WALKER, G., Politica española y comercio colonial, Barcelona, 1979.

(23) A.G.I. Impresos Antiguos (I.A) 23/26 Anóm. S.F. "Por parte de la ciudad de Cádiz informando como se la ha mandado de las conveniencias y utilidades que se seguirán de despachar en su puerto y bahía las Armadas y Flotas". 
Refiriéndose a la infraestructura de la zona afirma lo siguiente:

"Queriendo los navíos estar como metidos en una caja, no hay sino meterse al puntal estero de la Carraca y río del Puente Zuazo, donde pueden dar carena arrimados a la tierra y desde ella trabajar los oficiales, porque desde los dos fuertes del Puntal y Matagorda, que se están fabricando a estos sitios se hace un puerto cerrado capaz de tener en sí mil navios, por de gran porte que sean. Y queriéndose estar en el surgidero amarrándose bien están muy seguros de las tormentas y defendidos de los dichos dos fuertes que alcanzarán con las artillería, para que no se pueda alojar allí ninguna armada enemiga, y estarán puestos en defensa dentro de un año (24).

El texto indica también el interés que las autoridades militares ponían en estas obras. Sin duda guarda una gran relación con otro hecho importante: arbitrios de murallas, nuevos impuestos que se estaban otorgando a la ciudad para recoger los fondos de financiación de estas nuevas infraestructuras, etc. Sin duda, entre ello y los donativos monetarios que los grupos más adinerados e influyentes de la ciudad otorgaron desde el último decenio, del siglo XVI, constituyó un acto recíproco de ayuda y colaboración por parte de la Corona y la propia ciudad. Los planes de la Administración encontraron pronto apoyo inmediato de las instituciones gaditanas y hasta de la Capitanía General de Andalucía, pués sus objetivos incluían a todos los enclaves urbanos de la bahía como el Puerto de Santa María, a la que afectó de forma extraordinaria en aspectos políticos-militares y económicos (25).

\section{PATIÑo EN CADIZ}

El 11 de febrero de 1717 el nuevo Intendente-presidente llegó a Cádiz para llevar a la práctica una de las facetas consideradas como de las más importantes en las reformas practicadas a la Marina: la construcción del arsenal de la Carraca y la creación de la Escuela de Guardias Marinas, que fue llevada a cabo gracias a la colaboración del ministro con Antonio Gaztañeta (26). Su llegada a la ciudad pocos meses antes del nombramiento de Pez como presidente del Consejo de Indias fue considerado como un augurio de la Real Cédula de mayo de 1717 (27).

De su trabajo como Intendente-presidente en Cádiz entre 1717 y 1725 (salvo el intervalo de 1718 y 1721 , en su expedición a Italia y su misión en Flandes), podemos ver claramente cual fue su cometido: la reestructuración de una base naval que avanzara las reformas aplicadas al comercio y a la marina en los primeros años del siglo (28).

José Patiño fue enviado especialmente a una misión. Para ello aglutinó en su poder el control de muchas gestiones en toda la zona de la bahía. Con la capaci-

(24) Ibidem.

(25) GONZAlez Beltran, J.M., El Cabildo municipal del Puerto de Santa Mariá, Cádiz, 1993.

(26) RAVINA MARTIN, M., Op. Cit., p. 66.

(27) AHMC: L.A.C, Tomo 73 (1717), p. 85. (28) El mismo Patiño lo expone en su "Exposición Reservada" RODRIGUEZ VILI.A, A., Op. Cit.,
p. 23 . 
dad que le otorgaban sus títulos oficiales, el ministro gestionaba cuestiones mercantiles y de administración de la navegación, y también todo aquello referente a la adpatación del puerto gaditano a su nueva calidad de base naval y militar. De entre varias de las atribuciones que tuvo que afrontar estaba la de encargarse de supervisar la administración y financiación de las obras, pacto con asentistas y moderador entre autoridades civiles y militares de la zona (29).

Con respecto al Cabildo de la ciudad gaditana, había muchos factores que situaban a esta institución en contínua relación con la Administración central. Una de ellas fue la cuestión de los arbitrios. Como respondiendo a un plan provechoso, el Cabildo presionó a la Corona para mantener la construción de murallas y fortificaciones en la bahía. Se trataba, no obstante, de un plan estructurado y pensado desde la propia Administración., y ello lo prueba la existencia de una Planta de ingenieros y militares para la realización y gestión de dichas obras. Sin embargo, éstas se llevaban a cabo con una plena participación del Cabildo y algunas autoridades de la Capitanía General en el Puerto de Santa María que representó un papel importante para la Administración de Madrid con respecto a las cuestiones de apresto de Armadas y aprovisionamiento de materiales y víveres para la zona del puerto gaditano (30).

Con respecto a la propia financiación de las obras, el Cabildo llevaba la petición de impuestos específicos y la utilización de determinadas rentas de Aduanas, que en otras ocasiones fueron utilizadas también para extraer los donativos graciosos que la ciudad se veía obligada a ofrecer a la Real Hacienda (31).

Durante los primeros años del siglo XVIII el Cabildo Municipal gaditano pretendió aumentar las fuentes de ingresos de los arbitrios por medio de la creación de más impuestos especiales y que su administración y reparto corriese, no por delegados nombrados por la Corona, sino por personas elegidas especialmente por el Cabildo o presentadas por autoridades militares y civiles de la ciudad y que sirviesen a los intereses del propio municipio (32). En este caso (y no es nuestro cometido analizar con propiedad la problemática de la cuestión de los arbitrios de murallas, que a nuestro parecer necesita un estudio monográfico completo), la intervención de Patiño fue decisiva. Los miembros del Cabildo aprovecharon la llegada del ministro a la ciudad en 1717 y le ofrecieron su

(29) Las atribuciones generales que el Intendente tenía para toda la Peínsula: A.G.I. Libros Antiguos (L.A), s. XVIII, № 208, "Ordenanzas del 4 de julio de 1718 para el establecimiento e instrucciones de Intendentes, y para el Tesorero general, pagadores y contadores de los ejércitos y provincias. Por Orden de S.M. Impreso en 1735 por Juan de Aristía", Cfr: ABBAD, F. y OZANAM, D., Les Intendants espagnol du XVIIle siècle, Paris, 1992.

(30) GONZALEZ BELTRAN, J.M., Op. Cit., pp. 67-69. Una de las razones expuestas en el "Memorial de Vecinos", citado por el autor que data de 1727 hacía precisamente exposición de este papel llevado a cabo por el municipio porteño entonces perteneciente al Duque de Medinaceli, y que el 31 de mayo de 1729 fue incorporado a la Corona, junto a la Isla de León.

(31) AHMC. L.A.C, Tomo 73 (1717), pp. 263-269. Listas con la razón de las cantidades que se impusieron sobre el vino, la came y el vinagre para donativos a la Hacienda Real.

(32) AHMC. L.A.C, Tomo 74 (1718), Carta de Thomás de Idíaquez a Juan de Orta, 3 de junio de 1718 , pp. 224 y ss. Cabildo de 6 de julio de ese año propuso a Juan de Orta y a una plantilla de ingenieros y trabajadores formadas a gusto de las autoridades civiles y militares de la ciudad. La Corona nombró a Alberto Miençon como ingeniero jefe, a quien la ciudad rechazaba. 
apoyo en su misión de realizar el proyecto naval de la Corona. ¿Probablemente, el Cabildo y sus miembros sacaron algún provecho propio de la nueva coyuntura?. Cuando Patiño llegó a la ciudad hacía ya cincuenta años (desde 1680) que Cádiz era un objetivo prometedor como base de la Carrera de Indias de forma efectiva. Aún latía el tema del arrendamiento de las aduanas por Francisco Eminente y algunos otros casos en los que la oligarquía ciudadana participaba activamente. Sin duda vivian al acecho de los beneficios posibles de todo este entramado. Pero, al igual que no eludían posibles beneficios, estos grupos sociales no escatimaban en invertir en su propio terreno. Con los ingresos que de estos arbitrios se dedujeron se construyó toda la línea de murallas y fortificaciones, así como otras obras de infraestructura, tales como almacenes, cuarteles, Iugares para depósitos de bastimentos y pólvora, y todo tipo de albergues específicos para el apresto de la marina, incluyendo caminos de abastecimiento como la entrada al puente Zuazo. Los infórmes eran dados al Cabildo que ofrecía el permiso para la obra pero quienes revisaban y proponían éstas eran expertos relacionados con la administración de la ciudad y el ejército. José Patiño fue una de las personas que más trabajaron para la realización de estas obras. La prisa por llevar a buen término el proceso de adaptación de la bahía, por parte de la Secretaría del Despacho de Madrid está presente en el Cabildo de cuyas sesiones salía la decisión concreta de las obras, las cuales eran comentadas al Secretario D. Miguel Fernández Durán, así como la utilización y finalidad de cada construcción. José Patiño conservaba los planos de los proyectos generales para las mismas, y personalmente se encargaba de supervisar el nombramiento de ingenieros y maestres de obras, así como de dar el visto bueno a los materialés para la construcción y el costo de los mismos (33). El avituallamiento de la zona también contó con la ayuda de alguna que otra iniciativa particular: el Cabildo dió permiso el 6 de julio de 1718 a D. Luís de Ozío y Salazar para construir en la Isla de León una serie de hornos y almacenes para el apresto de la marina. Ozío fue uno de los principales colaboradores de Patiño en su calidad de proveedor de la Intendencia de Marina, recibiendo de él el consentimiento para ejecutar el acondicionamiento necesario en su propia casería en la Isla de León (34).

Por medio de Francisco de Varas y Valdés, entonces delegado por Patiño como presidente de la Contratación, se notificó a la Corona los gastos de estas obras ofrecidos por los comerciantes de la ciudad (35). Las labores de acondicionamiento de almacenes y hornos, así como de los puntos claves en el transporte de los avituallamientos en la zona de los caños e Isla de León (como era el puente Zuazo) se produjeron gracias a la aportación de algunos comerciantes gaditanos y miembros de la Real Armada como fueron : Barrios Leal, Ravaschiero, Pedro Infante y otros, quienes también colaboraron en la edificación del arrecife (36). Tal como la ciudad de Cádiz y su Cabildo manifestaron en esos años, tras el

(33) AHMC. L.A.C, Tomo 75 (1719) Cabildo 5 de febrero de 1719, p. 31.

(34) AHMC. L.A.C, Tomo 74 (1718) pp. 225 y ss.

(35) AHMC. L.A.C, Tomo 74 (1718), p. 232.

(36) AHMC. L.A.C, Tomo 74 (1718), p. 243 y v. 
problema del traslado de las instituciones estaban los intereses de los "fabricadores, Mareantes y dueños de navíos" (37).

Con el ministro Patiño en Cádiz, el Cabildo pedía su protección y consejo siempre que debía de decidir en algún tema referente al comercio y la aduana. La primera vez en la que se hace notar esta cuestión fue cuando la ciudad se acogió a la protección del Intendente, ante el Consejo de Hacienda, con motivo de haberse cumplido la la fecha de los arrendamientos de la aduana de la ciudad (38). Todavía no ha quedado aclarada cual fue la posición de Patiño con respecto al tema de las aduanas de Cádiz. Si pudiéramos profundizar con mayor cantidad de documentación en ello nos acercaríamos con más detalle al pensamiento económico que inspiraban las ideas del ministro. En el caso gaditano consideramos que Patiño, en parte influido por las ideas de su maestro el Almirante Pez, y en parte actuando por lo que le dictaba su conciencia y la preponderancia de sus súbditos gaditanos, medió ante la Administración para que la Corona otorgase alguna que otra prerrogativa favorable a la ciudad. El primer caso que conocemos fue la adjudicación a Cádiz del beneficio impuesto del 1 y tercio \% de Lonja (denominado Lonja de Infantes, y que antes cobraba el Consulado de Sevilla) para financiación de las obras de murallas. Ello se otorgó por una Real Facultad que dividía los beneficios de este pago entre el impuesto en sí que iba destinado a las obras del arrecife y una parte para la devolución de los empréstitos otorgados por la ciudad en los años de 1701 y 1702 (39). Y ello se continuó haciendo hasta después de 1726, cuando Patiño era ya Secretario de Estado.

El impuesto de Lonja de Infantes se debía de cobrar en la Aduana del Puerto de Santa María por el Superintendente de Rentas de esa ciudad D. Antonio Fernández de Castro. La rápida intervención de Patiño fue complementada con la fluidez de contactos que tenía el Cabildo y autoridades del municipio con el propio Secretario del Despacho de Guerra y Marina, entonces D. Miguel Fernández Durán (40). En ello vemos actuar de forma principal a la propia Capitanía General andaluza que colaboró a dos bandas tanto con Patiño como Intendente-presidente, como con el Cabildo gaditano. Como autoridades responsables de Cádiz y de sus gestiones marítimas y militares se veían involucradas activamente en todo aquello que se relacionase con el plan de fortificación plena de la plaza y el acondicionamiento naval de la misma, y también, por supuesto, de la financiación de estos trabajos. Sus actuaciones para pedirr impuestos destinados a las obras de murallas (en 1718, Juan Francisco Manrique y Arana y después Thomás de Idiaquez) fueron muy importantes, y se hicieron oir por parte de la propia Administración en Madrid. Junto con el Cabildo de la ciudad, actuaron como ele-

(37) AHMC, L.A.C, tomo 80 (1724), pp. 49-61. Los nombres de algunos de éstos aparecen en las gestiones del Cabildo a partir de 1721 y 1724 como autores de las cartas y memoriales ante el Almirante Pez con motivo de la revisión del problema del Tercio de Toneladas y las convocatorias de las Juntas de 1722 y 1723.

(38) AHMC. L.A.C, Tomo 74 (1718) Cabildo del 21 de enero de 1718, pp. 15 y 15v.

(39) AHMC. L.A.C, Tomo 75 (1719) Cabildo del 28 de febrero de 1719, pp. 64-77v.

(40) AHMC. L.A.C, Tomo 74 (1718), pp. 329 y ss. El Cabildo gaditano pidió representación directa con la Corona a través del favor de Fernández Durán, Secretario del Despacho. 8 de octubre de 1718 . 
mento de presión con el fin de aumentar los medios de financiación para estos fines, que según consta de sus memoriales y correspondencia, era un "deseo de la Corona" (41).

\section{LA VOZ DE JOSE PATIÑO EN LAS JUNTAS DE 1722 Y 1723}

Por lo que vemos de la práctica, la unión de intereses entre el Intendentepresidente y el Cabildo gaditano eran bastante afines. Pero irealmente eran los componentes del Cabildo un grupo de presión? ¿Hasta qué punto podemos diferir las peticiones y rápidas gestiones del Cabildo y de las autoridades militares del Puerto de Santa María de los trabajos de Patiño? ¿Fue Patiño ún verdadero promotor dé la construcción de la base naval gaditana, o sólo un enviado ante las solicitudes de los gaditanos y los propios planes de la Corona?

En la práctica, o lo que es lo mismo, viendo los documentos que señalan las actividades $y$ diligencias de unos y otros es dificil separar una cosa de otra. Por prudencia o por desconocimiento, el Cabildo y las autoridades de la Capitanía portuense sólo dejan ver sus deseos traducidos en contínuas solicitudes a la Corona sobre la necesidad del disfrute de impuestos especiales como es el caso visto del de Lonja de Infantes. Ello sólo era la necesidad de financiar las obras de ingeniería que la Corona deseaba llevar a cabo y finalizar lo más pronto posible. ¿La razón? el acondicionamiento de la bahía debía de ejecutarse en vista del futuro que le aguardaba, y no solamente por puras necesidades defensivas, sino estratégicas desde el punto de vista comercial y político de la propia Corona. Ofrecer a la ciudad el privilegio de la cabecera oficial de las flotas nos puede parecer un precio justo.

Sin duda que los miembros del Cabildo (todos ellos militares, comerciantes y negociantes relacionados con el sector naval) constituyeron un grupo de presión. Su actividadd económica y política se vió acrecentada en protagonismo con la facilidad de apoyo que le daban las autoridades militares de la Capitanía. Las obras marchaban con diligencia, la ciudad recibía de la Secretaría del Despacho de Madrid casi todo el disfrute de impuestos solicitados para la fábrica de murallas y fortificaciones, y además, recibía una atención especial por parte de las reformas de la Administración cuando en 1717 se instaló en la ciudad la "única" Intendencia que especificaba ser de Marina de la Península. Estaba claro que la preparación del puerto de Cádiz seguía un curso inevitable y sería la base naval y militar de la Carrera de Indias, se trasladase o no a la ciudad, el Tribunal de la Contratación. En 1725 las cosas caerian por su propio peso.

Pero además de su eficiencia administrativa y práctica como Intendentepresidente ¿qué papel tuvo Patiño en el entramado político que decidió el punto final de este proceso?

Cuando se convocó la Junta de 1722 Patiño envió desde Cádiz a la Corte todos los autos y cartas necesarias para que se tratasen todos los asuntos que solicitaba el presidente del Consejo, sin apartar ni uno, incluyendo el problema

(41) AHMC. L.A.C, Tomo 74 (1718) pp. 346 y ss. Carta del Capitán General de Andalucía al Cabildo para aumentar los impuestos de Lonja y otros sobre el cargue para Indias con el fin de financiar las obras de fortificación, 20 de octubre de 1718. 
de astilleros, maestranzas, pertrechos para la Armada, etc. En estas juntas la opinión de Patiño pudo tener mucha importancia ya que en cierto modo se consideraba continuador de la obra del Almirante, y su heredero político a partir de 1725 . Su voz en la Junta de Madrid de diciembre de 1723 (una vez desaparecido el Almirante) declara por fin, y de forma escrita las ideas personales del ministro. En esta nueva sesión, la segunda que se realizó sobre el traslado de tribunales, el voto del Intendente-presidente es significativo. En primer lugar se refirió a cuestiones puramente técnicas, como fue el problema de los sondeos practicados a la barra de Sanlúcar realizados hasta ese momento, y algunas ideas no muy extensas sobre los problemas del comercio con América que nos acerca a la razón de algunas de las innovaciones introducidas por Patiño en los años anteriores a 1722 y que retomaría como Secretario de Estado en 1726. En la nueva sesión de los debates, Patiño solamente ve como novedoso y práctico la investigación y sondeeo de la barra de Sanlúcar realizada por Gaztañeta y que confirmaba los problemas que presentaba la navegación en esa zona (42). Patiño se mostró disconforme con el resultado del resto de los informes mandados hacer por Sevilla posteriormente afirmando que éstos estaban manipulados o realizados por personas inexpertas en la materia que incluian omisiones de hechos conocidos, como, por ejemplo, que el fondo de la barra era de piedra, y algunos otros errores intencionados (43).

Pero a nuestro parecer, el punto más interesante de la postura de Patiño es su intento de aclarar su opinión acerca de dos puntos. Curiosamente, el ministro no hace mención directa de la habilitación del puerto de Cádiz para los aprestos y despachos de Armadas. Ello nos puede hacer creer una intención de no comprometerse en sus relaciones con las autoridades militares de la zona y con las personas de la ciudad que mantenían ya negocios importantes en la marina.

Los dos puntos que menciona Patiño en su voto son: Aclarar si es navegable o no la barra de Sanlúcar, y si deben subsistir los tribunales del comercio en Cádiz o Sevilla como necesaria consecuencia de ello. La opinión del ministro con respecto a ello fue la siguiente:

El polémico tema de la navegabilidad de la barra se dividía en dos cuestiones o principios. El primero es referente a las calidades y características que debían de contemplar la construcción de barcos; y el segundo punto hacia referencia a los múltiples sondeos practicados y sobre la credibilidad que debían de tener las personas encargadas de los mismos. Para Patiño, al igual que para Gaztañeta y Pez, la barra era peligrosa por su formación geológica, así como la navegación río arriba, era físicamente imposible para una nao de alto calado transitar por ella, y moralmente imposible también por riesgo de naufragio a las embarcaciones ligeras o de pequeño buque. Los múltiples memoriales a la Corona arguyendo esta cuestión se hicieron por parte de negociantes y navegantes debido al miedo que

(42) A.G.I. ARRIBADAS 10, El sondeo realizado por D. Antonio Gaztañeta Yturrivalzaga por orden expresa de D. Andrés de Pez y copia de la Real Orden del 12 de marzo de 1722. La relaciôn entre ambos es muy significativa ya que el Almirante Gaztañeta estaba muy relacionado con los proyectos de reformas de la Marina.

(43) A.G.I. ARRIBADAS 10. Voto del ministro Josée Patiño en las Juntas de Diciembre de 1723. Passim. 
este hecho causaba con motivos justificados. Según el ministro, de todas las barras del mundo, la barra de Sanlúcar era la mâs peligrosa y como contrapartida a ello justificaba como la bahía y puerto de Cádiz:

“...no tiene parangón en otra parte de Europa y que son envidiadas de cualquiera nacional e inteligente, las circunstancias de su seguridad, surgidero y carenero en los caños que se extienden tietra adentro hasta el puente Zuazo de forma que viene a tener las dos estimables calidades que rara vez junta una provincia de una bahía abierta para la facilidad de su entrada, y salida de puerto cerrado en la estreches de sus caños, en el resguardo de sus castillos o fuertes y en el abrigo de los vientos" (44).

Con respecto a la idea de reducir la capacidad de los bajeles, Patiño se muestra disconforme, indicando claramente que esto era altamente perjudicial para el comercio. Evidentemente, el ministro está defendiendo los intereses, no sólo de comerciantes y hombres de negocios en general, sino particularmente de los fabricantes de navíos, cuya participación mediante la capacidad de carga de sus buques era uno de los factores más importantes en la reunión de las flotas para América. Anteriormente hubo muchas personas relacionadas con la Casa de la Contratación y la navegación a América que estaban a favor de estas ideas nuevamente esbozadas por Patiño sobre la imposibilidad de acometer la subida de la barra hasta Sevilla y la inconveniencia de que se construyesen navíos de pequeños cálado (45).

Como consecuencia de estos problemas, la única solución era la permanencia en Cádiz de la Casa de la Contratación y la Intendencia (en ningún momento se nombra al Consulado). La estancia del Tribunal que regía el monopolio en esta ciudad se explicaba, según Patiño, no sólo por los problemas que traía la barra, sino también porque donde estaba la base naval más importante del país debía de estar la terminal de las flotas y galeones, y allí debían ser aprestadas, despachadas y recibidas a su regreso. Además de ello estas cuestiones debían de estar interrelacionadas con el origen de las providencias gubernativas y de justicia que otorgaban estos tribunales, tanto "para el bien universal de los traficantes y comerciantes como para los Reales intereses", así como evitar los abusos y daños que la distancia de su residencia anterior en Sevilla había provocado (46). Además incidía en la necesidad de que se mantenga unida a la Administración central mediante los encargos de su presidente. Justificados motivos, pues este tipo de organismos institucionales del comercio ( $\mathrm{y}$ en ello hace referencia a los autores de Derecho Mercantil de la época) debían de residir en las zonas donde propiamente sea su territorio, donde sea más fácil la administración de justicia, próximos los recursos y menos costosas las diligencias que este trabajo producía. Está

(44) Ibidem.

(45) A.G.I. ARRIBADAS 10. Patiño enumera a una serie de personas expertas para afianzar sus ideas: Marqués de Fuente de Sol, Gonzálo de Córdoba, Fernando de Villegas, José De Veitia (antiguos ministros de la Contratación), General Príncipe de Montesacro, Almirante Antonio de Echevarri, Capitán Juan de Pontejos Salmón, etc.

(46) Passim. 
claro, de nuevo, el pragmatismo del ministro, conocedor de su trabajo administrativo después de su experiencia en el puerto gaditano y que además recogía las ideas más competentes recibidas tanto de sus superiores en Madrid (Pez, Bergeyck y Tinajero), como de los negociantes y autoridades locales con las que estaba acostumbrado a relacionarse.

En los temas relacionados con el desarrollo de los negocios mercantiles, Patiño se muestra ávido de mantener el monopolio de la Corona, pero también lograr una mayor relación entre los propios negociantes implicados en ello. ¿Aparece aquí intrínseca la idea de la Compañía?. El ministro exponía su deseo de que existiese una perfecta correlación entre los negocios navales, los intereses mercantiles particulares, y las gestiones técnico-administrativas del puerto. En ello hace especial hincapié en el "capital social" de los negocios, los navíos. Así, desde que las Flotas y Galeones o navíos sueltos se divisaban en el horizonte de la bahía comenzaban a disponerse las providencias que debían de dar estos tribunales para el resguardo de los intereses de la Administración y de los propios comerciantes, que continúan hasta su entero alijo y entrega a sus dueños de los caudales, como de arbitrar entre los pleitos que sucedian entre los particulares por motivos de fletes, seguros marítimos, etc. Y posteriormente a ello, se producía el desarme de los navíos, el pago a las "gentes de mar", y a los fabricantes de buques, las carenas, las visitas, las remisiones de los caudales a la Real Hacienda, las organizaciones de navíos sueltos o Avisos, etc. El ministro volvía a incidir en el especial papel que aún tenía la antigua Audiencia de la Contratación de Sevilla, y en la necesidad de que el presidente interviniera como árbitro en lo jurisdiccional de los problemas que en el puerto causaban los registros de tripulaciones, derechos de navios, fletamientos, etc (47).

Una de las partes más polémicas sobre la actuación de Patiño es la referente la cuestión del asentamiento de los comerciantes en la ciudad. En un principio, se dijo, era Sevilla el lugar de asentamiento mercantil y en un momento determinado se pasaron a Cádiz, luego se sobreentiente la realidad de la decadencia sufrida por Sevilla en un momento determinado. Patiño afirma que esto no es pretexto para esgrimir argumentos contra Cádiz. Afirma además que la existencia de la Diputación y el Consulado de Sevilla es "quasi inutil" porque con respecto a las cuestiones jurisdiccionales ya estaban las Justicias ordinarias de cada ciudad, y para los problemas del comercio con Indias estaba el Tribunal de la Contratación, que además de ser más imparcial, atendía a estos asuntos en el momento justo que debía hacerse: en el despacho de las flotas y galeones desde el puerto.

Patiño también se mantiene en la misma postura refiriéndose a los cargadores y cosecheros de Sevilla y Cádiz. En ello se intuye la diferenciación que hace con respecto a la terminología usada para los de una y otra ciudad: Denomina "cargadores y cosecheros" a los comerciantes sevillanos, mientras se dirige a los gaditanos como "cargadores aunque no todos sean cosecheros" y admite que éstos últimos emplean más caudales en el tráfico. Sin embargo, Patiño no se manifiesta claramente sobre la cuestión de los extranjeros en Cádiz y apunta la realidad de que no es solamente un problema de Cádiz, y

(47) Ibidem. 
que gran parte de los comerciantes sevillanos también lo son, o al menos descendientes de éstos (48).

A pesar de que posteriormente, Patiño se decantó a favor de ejercer un mayor control sobre estas actividades de extranjeros, por el momento su opinión acerca de este tema no se ve muy clara.

Después de la finalización de estos debates en el año 1723 la indecisión que afectó al gabinete de Felipe V, produjo que muchos de los problemas suscitados durante los debates quedasen sin aclarar, decidiéndose en 1725 la restitución de los tribunales a Sevilla, aunque no la anulación del proceso que se estaba llevando a cabo en el puerto gaditano (49). Sin embargo, una nueva coyuntura favorable vino a producir la supresión de este decreto: los nuevos cambios producidos por la inestabilidad política y la aparición momentánea de Ripperdá, quien firmó en Madrid el 31 de diciembre de ese año una carta expresando que el Tribunal no debía regresar a Sevilla (50). A pesar de las peticiones del Consulado de Sevilla, la decisión por Cádiz estaba tomada en la Corte. Hay motivos claros por los que no fueron escuchadas las súplicas del Consulado: Aparte del envío del Memorial de Herrera, que por fin denota claramente la postura del Cabildo gaditano ante la Administración, la subida de Patiño a la Secretaria del Despacho de Madrid, daba paso, de alguna manera, a la puesta en práctica definitiva de una serie de ideas y proyectos que, ya en 1726 (con la creación de los Departamentos Marítimos) era un hecho imparable. Una carta enviada al nuevo Secretario de Estado por el Cabildo de la ciudad denota la particular predilección que el ministro tenía por mantener la preparación del puerto de Cádiz (51).

(48) Ibidem.

(49) A.G.I. INDIFERENTE GENERAL, 2039, Decreto del 31 de septiembre de 1725. (copia).

(50) A.G.I.INDIFERENTE GENERAL, 2021, copia de carta notificando la decisión a Francisco de Varas y Valdés. 31 de diciembre de 1725. AHMC. L.A.C, Tomo 81 (1725) pp. 511-539. Según el Cabildo de Cádiz fueron Francisco Manuel de Herrera y Ripperdá los que consiguieron la anulación del decreto. Los enemigos de Patiño relacionaban al ministro con el Duque.

(51) A.G.I. INDIFERENTE GENERAL, 2300. Copia de carta del 2 de junio de 1726 firmada en Cádiz por Pedro Baeza, Antonio Alvárez de Bohorquez (gobernador de la plaza y posteriormente Capitán General de Andalucía), Francisco Joseph Roldán y Manuel Pérez Angulo, dirigida a Patiño como Secretario de Estado. 